\title{
Intraurethral immunoglobulin in the treatment of non-specific urethritis
}

\author{
D W SPELMAN* AND D BRADFORD† \\ From the ${ }^{*}$ Fairfield Infectious Diseases Hospital, Fairfield, Victoria, and the + Communicable Diseases \\ Centre, Melbourne, Australia
}

SUMMARY We report on a 25 year old man with agammaglobulinaemia and refractory nonspecific urethritis. After seven months of unsuccessful antibiotic treatment, his symptoms responded to the administration of intraurethral immunoglobulin.

\section{Introduction}

Non-specific urethritis (NSU) is a common sexually transmitted disease. Agammaglobulinaemia is an unusual congenital immunodeficiency. As far as we know the combination of NSU and agammaglobulinaemia and the use of intraurethral immunoglobulin have not previously been reported.

\section{Case Report}

In October 1981 a 25 year old man was referred from the Communicable Diseases Centre(CDC), Melbourne to Fairfield Infectious Diseases Hospital, Melbourne, with recurrent and persistent NSU of 20 months' duration.

\section{History \\ He had a history of agammaglobulinaemia, diagnosed initially at two years of age, which required regular immunoglobulin replacement. Until the age of 10 years he suffered from chronic sinusitis, recurrent episodes of respiratory tract infection, conjunctivitis, and diarrhoea. Through his teenage years his health was generally better. Since 1976 he had had single episodes of herpes zoster, giardiasis, and pneumonia. From 1975 he had given himself a weekly $5 \mathrm{ml}$ intra- muscular injection of normal immunoglobulin (human). \\ He first presented to CDC in February 1980 with a urethral discharge. Gram negative diplococci were seen on a stained smear of the discharge, but Neisseria gonorrhoeae was not cultured. He was treated with a single dose of amoxycillin plus probenecid, and one week later received a 10 day \\ Address for reprints: Dr D W Spelman, Department of Bacteriology, Alfred Hospital, Commercial Road, Prahran, Victoria, Australia \\ Accepted for publication 1 August 1983}

course of tetracycline $(0.5 \mathrm{~g}$ four times a day) for symptoms of postgonococcal urethritis. This resulted in complete resolution of his symptoms. Samples for Chlamydia trachomatis culture were not taken on his initial visit. On two further occasions during 1980 (in April and October) he developed a urethral discharge, gonococcal cultures gave negative results, and he was treated for NSU with tetracycline. With the final episode, a second course of tetracycline was needed.

He next presented to CDC with urethral discharge and dysuria in July 1981. A Gram stained smear of the discharge contained numerous polymorphonuclear leucocytes and because of a possible contact with gonorrhoea he was initially given amoxycillin plus probenecid, followed by a seven day course of erythromycin $(0.5 \mathrm{~g}$ three times a day). This resulted in only partial relief of his symptoms. He subsequently received five further courses of antibiotics (co-trimoxazole, tetracycline, tinidazole, doxycycline, and metronidazole), but symptoms persisted. During this time his girlfriend was treated with a 10 day course of tetracycline. She was asymptomatic and no cultures were taken from her.

\section{Examination}

When he was referred to Fairfield Hospital in October 1981, his symptoms had been present for three months. He had had one steady sexual partner during 1981, but had not had sexual intercourse for at least six weeks before his referral. On examination the only abnormal findings were teeth stained by many courses of tetracycline and an inflamed external urinary meatus. Rectal examination was normal. Microscopical examination of a Gram stained smear of the urethral discharge again showed numerous polymorphonuclear leucocytes. Cultures for $N$ gonorrhoeae, $C$ trachomatis and herpes simplex virus gave negative results. Culture of urine 
also gave negative results. Immunoglobulin measurement showed a low concentration of IgG only.

\section{Management}

Over the next four months he received prolonged courses of erythromycin, metronidazole, and doxycycline, and finally co-trimoxazole; he did not have sexual intercourse during this time. Each antibiotic regimen gave only partial relief of his symptoms. Repeated bacterial and chlamydial cultures of the urethral discharge gave negative results. He also complained of intermittent low grade conjunctivitis late in 1981.

In February 1982 urological pandendoscopy disclosed florid anterior urethritis, and he was admitted to hospital. Using a syringe and urinary nozzle, intraurethral irrigation with normal human immunoglobulin (also called human gammaglobulin) at a dose of $1 \mathrm{ml}$ three times daily was started. On the same day monthly intravenous infusions of $200 \mathrm{ml}$ of normal immunoglobulin in place of weekly intramuscular injections were started.

The patient reported immediate symptomatic relief following intraurethral irrigation with immunoglobulin. He continued this treatment for five weeks and had no urethral symptoms for the following five months. His symptoms returned, however, after resumption of sexual intercourse with a new partner. At that time Ureaplasma urealyticum was cultured from a urethral swab, urine, and also from an eye swab. This was the first occasion on which specimens from this patient had been cultured for ureaplasma. Intraurethral immunoglobulin irrigations were resumed and a course of doxycycline prescribed. His symptoms completely resolved, and ureaplasma cultures were subsequently negative.

\section{Discussion}

A feature of agammaglobulinaemia is recurrent mucosal infections, especially in the respiratory tract. Urinary tract infections are uncommon, ${ }^{1}$ and as far as we know NSU in such a patient has not previously been described. We do not know the exact role, if any, of agammaglobulinaemia in the persistence of this patient's symptoms. The relative importance of circulating immunoglobulins and mucosal immunity in combating NSU is also unknown. The standard immunoglobulin, used in this case for intraurethral irrigation, contains IgG antibodies only.

In the case reported here, both the intraurethral irrigations and the monthly intravenous infusions started at the same time. The latter resulted in higher serum trough concentrations of $\mathrm{IgG}(25 \mathrm{IU} / \mathrm{ml})$ than those achieved with weekly intramuscular injections $(16 \mathrm{IU} / \mathrm{ml})$. It is therefore possible that the intravenous infusions had a therapeutic role. The intraurethral immunoglobulin did, however, give impressive immediate symptomatic relief.

A causative organism had not been isolated before the start of intraurethral irrigations, and when ureaplasma was isolated his symptoms had recurred after a symptom free period of five months despite monthly intravenous immunoglobulin. At that time his symptoms resolved with reinstitution of intraurethral immunoglobulin plus doxycycline. Thus intraurethral immunoglobulin appeared to be effective in this agammaglobulinaemic patient with symptoms of refractory NSU.

\section{Reference}

1. Grieco MH. Infections in the Abnormal Host. 1st. ed. New York: Yorke Medical Books, 1980. p. 142. 